ISSN: 2174-5609

DOI. https://doi.org/10.14198/INTURI2022.23.7

Cita bibliográfica: Menor Campos, A., Hidalgo Fernández, A., López Felipe, T. y Jara Alba, C. (2022). Gastronomía local, cultura y turismo en Ciudades Patrimonio de la Humanidad: el comportamiento del turista extranjero. Investigaciones Turísticas (23), pp. 140-161 https://doi.org/10.14198/INTURI2022.23.7

\title{
Gastronomía local, cultura y turismo en Ciudades Patrimonio de la Humanidad: el comportamiento del turista extranjero
}

Local gastronomy, culture, and tourism in World Heritage Cities: the foreign tourist behaviour

Antonio Menor-Campos (iD, Universidad de Córdoba, España antonio.menor@uco.es

Amalia Hidalgo-Fernández (iD, Universidad de Córdoba, España ahidalgo@uco.es

Teresa López-Felipe (iD, Universidad de Córdoba, España mtlopez@uco.es

Carol Jara-Alba (iD, Universidad Casagrande de Guayaquil, Ecuador cjara@casagrande.edu.ec

\section{RESUMEN}

Actualmente, la gastronomía se muestra como un componente fundamental para los turistas enel descubrimiento de la herencia cultural del lugar. Ello implica que los placeres culinarios se conviertan en motivaciones determinantes en la elección del destino, y, al mismo tiempo, se manifiesten como un factor explicativo esencial de la satisfacción que experimenten los turistas. El objetivo de este trabajo es analizar las experiencias que, en el ámbito de la gastronomía, tienen los visitantes extranjeros en una ciudad Patrimonio de la Humanidad, como es el caso dela ciudad de Córdoba. Los resultados obtenidos permiten, en base a las percepciones sobre las experiencias gastronómicas en la ciudad, clasificar a los turistas en tres grupos que se han denominado supervivientes, disfrutadores y experimentadores. Los resultados sugieren que la experiencia gastronómica del turista extranjero varía en función de la actitud del visitante haciala gastronomía en los viajes.

Palabras clave: Gastronomía; turismo; Córdoba; segmentación; experiencia gastronómica. 


\section{ABSTRACT}

Currently, gastronomy constitutes a fundamental component for tourists when discovering the cultural heritage of a destination. This implies that culinary pleasures become decisive motivations in the choice of destination, and, at the same time, manifest as an essential explanatory factor of the satisfaction experienced by tourists. This study seeks to analyse the gastronomic experiences of foreign visitors to World Heritage Cities, such as the city of Córdoba. Based on perceptions of gastronomic experiences in the city, the results enable us to classify tourists into three groups that have been called survivors, enjoyers and experimenters. The findings suggest that the gastronomic experience of the foreign tourist varies depending on the attitude of the visitor towards gastronomy in the trips.

Key words: Gastronomy; tourism; Córdoba; segmentation; gastronomic experience.

\section{INTRODUCCIÓN}

La satisfacción con el destino por parte de los turistas se ve cada vez más condicionada por la valoración de la experiencia gastronómica tenida en el mismo y, especialmente, en lugares con un gran contenido teórico histórico-cultural. En este trabajo se lleva a cabo una segmentación de los turistas extranjeros apoyada en la gastronomía. Posteriormente, se realiza un análisis, basado en la segmentación efectuada, de la experiencia gastronómica en un destino patrimonial de primer orden como es la ciudad de Córdoba. En la actualidad cuenta con cuatro inscripciones de la UNESCO, de las cuales tres lo son como Patrimonio de la Humanidad (incluyéndose aquí la Mezquita-Catedral, la zona de la Judería y el conjunto arqueológico de Medina Azahara), y la restante como Patrimonio Inmaterial de la Humanidad (Fiesta de los Patios).

La gastronomía local pone de manifiesto valores que engloban tanto los aspectos puramente fisiológicos, es decir, aquellos que derivan de la necesidad de cualquier individuo de alimentarse, sea o no turista, como los relacionados con la comprensión de la historia, las costumbres ancestrales o las relaciones sociales de un destino. En este sentido, conviene estudiar y analizar los diferentes segmentos de turistas existentes según su interrelación con la gastronomía del sitio visitado, conociendo sus motivaciones y percepciones en relación con la gastronomía, variable de atracción y lealtad turística (Hjalager, 2004; Pesonen, Komppula, Kronenberg y Peters, 2011). Las posibilidades que determina la experiencia gastronómica son múltiples, pudiendo jugar desde un simple papel auxiliar y logístico del viaje, hasta constituir un factor clave en el desarrollo de una experiencia turística e, incluso, llegar a ser un elemento determinante en la elección del destino (Basil y Basil, 2009; López-Guzmán, Uribe-Lotero, Pérez-Gálvez y Ríos-Rivera, 2017). Asimismo, recientemente se está analizando una nueva relación entre el turismo y la gastronomía, de la mano de viajeros muy exclusivos, como es el caso de los chefs, que intentan encontrar en sus desplazamientos novedades en cuanto a técnicas culinarias, productos, texturas, sabores, etc. para aplicarlo posteriormente como nuevo conocimiento en sus propios restaurantes, convirtiendo la gastronomía de los destinos visitados en fuente de innovación para sus desarrollos culinarios.

La gastronomía también contribuye a mejorar la economía de los diferentes destinos, especialmente la de países en vías de desarrollo (Mgonje et al., 2016). En este sentido, el turismo gastronómico se erige como un magnífico dinamizador de algunos destinos, 
propiciando que el valor agregado de los productos permanezca allí donde son generados. Sobre esta base, diversas investigaciones están procediendo al estudio del turismo y de la gastronomía bajo tres enfoques diferentes: (1) desde el punto de vista del consumidor, (2) desde el punto de vista del productor y (3) considerando la aportación al destino a través de un desarrollo económico-social (Anderson, Mossberg y Thekelsen, 2017).

Este trabajo pretende contribuir a la literatura académica existente sobre las experiencias gastronómicas de los viajeros extranjeros. El objetivo fundamental es el análisis, a través de la segmentación de los turistas, del interés y de las motivaciones de los visitantes extranjeros en relación con la gastronomía en la ciudad de Córdoba.

Estudiar el turismo gastronómico supone analizar el comportamiento del turista. Un viajero que no manifieste un especial interés por la gastronomía local o que no viaja con la intención de degustarla, se comporta como un visitante que sólo busca alimentarse durante su estancia en el destino elegido. Por el contrario, los turistas interesados por la gastronomía viajan teniendo como motivación, bien principal, bien secundaria, el conocimiento de una gastronomía diferente, el aprendizaje sobre ésta, el relajarse saboreando la cocina típica local o el enriquecimiento cultural. Es decir, buscando una experiencia gastronómica auténtica (López-Guzmán, Hernández-Mogollón y Di Clemente, 2014).

Las investigaciones llevadas a cabo sobre esta materia revelan dos aspectos muy importantes (Fields, 2002; Hall et al., 2003): en primer lugar, los turistas que se muestran interesados por la gastronomía local suelen disponer de una mayor capacidad de gasto; en segundo lugar, los turistas que viajan por razones gastronómicas suelen ser mucho más exigentes con la calidad y con la autenticidad de la gastronomía local degustada.

\section{REVISIÓN DE LA LITERATURA}

\subsection{Concepto de turismo gastronómico}

Para Hall et al. (2003), el turismo gastronómico consiste en la visita a productores primarios o secundarios de alimentos, festivales gastronómicos, restaurantes y a otros lugares con el objetivo de saborear o tener experiencias gastronómicas con productos locales o regionales. Existe una amplia discusión en la literatura científica sobre el concepto de turismo gastronómico. Así, en inglés han surgido diferentes términos como food and wine tourism, tasting tourism, gourmet tourism, culinary tourism, food tourism o gastronomic tourism (Ellis, Park, Kim y Yeoman, 2018).

Según Ellis et al. (2018) el concepto de turismo gastronómico se puede definir desde dos perspectivas. La primera, está centrada en el propio turista y aborda su actividad y motivación. La segunda, se centra en el destino y analiza cuatro cuestiones diferentes: las diversas tipologías de turismo, los recursos en el destino, los productos turísticos y la promoción del destino sobre la base de la gastronomía.

Por otro lado, el concepto de turismo gastronómico se define a través de cinco temas (Ellis et al., 2018): motivación, cultura, autenticidad, gestión y marketing, y destino. De estos conceptos, el primero que define al turismo gastronómico es la motivación, que abarca aspectos como la experiencia o cuestiones relacionadas con la salud (Kim, Eves y Scarles, 2013). El segundo concepto sería la cultura y, con referencia a este punto, hay que destacar que la gastronomía forma parte de la herencia cultural de la comunidad local (López-Guzmán 
et al., 2017). La autenticidad ocupa el tercer lugar y representa un aspecto básico para el desarrollo de la gastronomía de un lugar y un motor para la promoción del turismo. En cuarto lugar, la gestión y el marketing del lugar comprenden aspectos básicos como el comportamiento del consumidor; y, por último, en quinto lugar, el análisis de la relación existente entre el destino y la gastronomía.

\subsection{Segmentación de los turistas en base a la gastronomía}

La segmentación de los turistas que visitan un destino es un elemento básico para el desarrollo de una correcta gestión de éste, tanto por empresas privadas como por gestores públicos. Ello puede facilitar el desarrollo de productos turísticos específicos orientados a la satisfacción de las diferentes necesidades que afecten a los turistas de cada uno de los segmentos obtenidos. Así, en los análisis de segmentación se suelen incluir variables como las motivaciones, los estilos de vida o los perfiles sociodemográficos, haciendo posible la identificación de grupos de turistas con características homogéneas, dada la complejidad de individuos que visitan un destino interesados en su gastronomía (Levitt, Zhang, DiPietro y Meng, 2017; Ko, Kang, Kan y Lee, 2018) y también los grados de interés que muestran los visitantes por la misma (Kivela y Crotts, 2005).

Cada segmento de turistas es, generalmente, considerado como un grupo con intereses, perspectivas y necesidades similares, y unas características sociodemográficas parecidas. Una información fundamental para el desarrollo de operaciones de promoción y la creación de productos turísticos. Kivela y Crotts (2005) señalan la necesidad de segmentar a los turistas que visitan un determinado destino según la valoración que realizan de su gastronomía, siendo fundamental para ello dar respuesta a tres cuestiones: primera, la determinación de cuáles son los turistas más interesados en la gastronomía y cuáles son sus principales características socio-demográficas; segunda, la delimitación de qué segmentos de turistas ofrecen un mayor retorno de la inversión en función de su capacidad económica; y tercera, el análisis del porqué estos turistas adquieren unos determinados productos gastronómicos en vez de otros. Por tanto, la segmentación del turista es esencial para la vertebración de las políticas turísticas en los destinos y para que las empresas, privadas y públicas, doten al destino de productos que permitan una mayor satisfacción a cada una de las tipologías de turistas.

En cuanto a la segmentación de los gastroturistas cabe destacar, entre otros, los estudios de Kivela y Crotts (2005), McKercher, Okumus y Okumus (2008), Björk y KauppinenRäisänen (2016) y Robinson, Getz y Donilcar (2018). Estas investigaciones ofrecen evidencias empíricas de que los turistas más atraídos por la gastronomía del lugar de destino generalmente son los que poseen un mayor poder adquisitivo. Asimismo, estas investigaciones señalan que esta tipología de turistas presenta un mayor grado de exigencia en relación con aspectos tales como la singularidad de la cocina local o su calidad. Los estudios científicos centrados en la segmentación del turismo gastronómico se agrupan principalmente en tres bloques: análisis de los destinos turísticos (Kivela y Crotts, 2005; McKercher et al., 2008; López-Guzmán et al., 2017), estudio de los festivales gastronómicos (Kim, Ducan y Chang, 2015, López-Guzmán et al., 2017) y análisis de los mercados gastronómicos (CrespiVallbona y Domínguez Pérez, 2016).

Por su parte, Kivela y Crotts (2005), clasifican los turistas en función de tres aspectos esenciales: el conocimiento gastronómico que presenta el turista; el interés por la 
gastronomía como elemento determinante en la toma de la decisión de viajar; y la experiencia gastronómica que el visitante ha obtenido. Estos autores diseñan un modelo de segmentación del turista gastronómico basado en las respuestas de los turistas a los interrogantes planteados, que contempla dos grupos: un primer grupo formado por turistas con un limitado interés por la gastronomía local y más interesados por otros recursos del destino, y un segundo grupo que incluye a aquellos turistas interesados por aprender y degustar la gastronomía del destino.

McKercher et al. (2008) realizan la segmentación de los viajeros empleando tres cuestiones: la primera estudia la autoconsideración por parte del propio turista como un turista gastronómico; la segunda analiza si el turista se define a sí mismo como interesado en conocer y probar la variedad gastronómica del lugar de destino; y la tercera versa sobre si el turista considera la gastronomía de un lugar concreto como una motivación básica al tomar la decisión de visitar un destino. En función de la respuesta dada a los interrogantes anteriores, se establecen cinco categorías o segmentos de turistas según su interés por la gastronomía: non culinary tourist, unlikely culinary tourist, possible culinary tourist, likely culinary tourist, and definite culinary tourist.

Thompson y Prideaux (2009), siguiendo el modelo de Hall y Sharples (2003), establecen cuatro argumentos para la segmentación de los turistas en relación con la gastronomía: primero, si visitan el área geográfica interesados por la gastronomía y por el vino; segundo, si consideran la gastronomía y el vino como una de las razones para la visita al destino; tercero, si estarían dispuestos a adquirir productos gastronómicos locales; y cuarto, otras motivaciones no gastronómicas para visitar el lugar. Y sobre la base de estas cuatro cuestiones, Thompson y Prideaux (2009) determinan tres grupos de turistas: food and wine tourists, undecided tourists y not interested tourists.

Björk y Kauppinen-Räisänen (2016) presentan una categorización de los turistas basándose en tres cuestiones. El primero es analizar la influencia de la búsqueda de nuevas experiencias gastronómicas por parte de los turistas como elemento motivador al adoptar la decisión de realizar el viaje. El segundo es estudiar cómo influye la gastronomía al optar por un determinado destino. Y el tercero es analizar la relación que existe entre la gastronomía y la satisfacción del visitante con ese lugar. Con estas tres cuestiones, Björk y KauppinenRäisänen (2016) segmentan a los turistas en tres grandes grupos. El primer grupo, denominado experiencers, incluye a aquellos turistas para los que la gastronomía es una parte fundamental de su viaje. El segundo grupo, denominado enjoyers, agrupa a aquellos turistas que muestran un cierto interés en el disfrute de la gastronomía y de las experiencias culinarias. El tercer grupo, denominado survivors, comprende a aquellos turistas que no consideran la gastronomía como un aspecto importante en su viaje.

Finalmente, Robinson et al. (2018) realiza una segmentación basada en la aportación de los turistas a las diferentes actividades ligadas con la gastronomía del destino. En función de estas cuestiones, estos autores agrupan a los turistas en dos grandes grupos: eruditos e ignorantes, diferenciándose en el gasto realizado por los turistas sobre aspectos relacionados con la gastronomía en el lugar de destino.

Por otra parte, existen diferentes estudios de carácter exploratorio, relacionados con el gastroturismo, que segmentan los visitantes en función de su nacionalidad, diferenciando entre turistas nacionales y extranjeros. En esta línea, Alonso, Fraser y Cohen (2007) presentan 
los resultados de una investigación sobre los perfiles sociodemográficos de los enoturistas en Nueva Zelanda segmentados por nacionalidad. Asimismo, y en el campo del turismo gastronómico, Nam y Lee (2011) presenta un estudio sobre la satisfacción de los turistas extranjeros en los restaurantes tradicionales coreanos. Por su parte, Horng, Liu, Chou y Tsai (2012) analizan la percepción de la marca gastronómica de ese lugar por parte de los turistas extranjeros.

La presente investigación se fundamenta en el modelo más reciente de segmentación de los turistas gastronómicos que aporta Björk y Kauppinen-Räisänen (2016). Este modelo se basa en tres premisas para realizar esta segmentación: primera, el interés por la gastronomía por parte de los turistas; segunda, la gastronomía como motivo para viajar; y tercera, la experiencia gastronómica como elemento básico para elegir un destino (Björk y KauppinenRäisänen, 2016).

De acuerdo con la revisión de la literatura, contrastaremos la siguiente hipótesis:

H1: Los turistas muestran diferentes actitudes hacia la gastronomía como variable de interés en la elección de un destino (Björk y Kauppinen-Räisänen, 2016).

\subsection{Perfil sociodemográfico de los gastroturistas}

El análisis del perfil sociodemográfico de los viajeros vinculado a la gastronomía se circunscribe en el marco de estudio de los patrones de consumo culinario (Park, 2017; Robinson et al., 2018). Ignatov y Smith (2006) señalan que el segmento de turistas para los que la gastronomía es un componente relevante en la elección del destino abarca aquellos que tienen una edad en torno a los 45 años, un nivel de formación alto y un nivel de renta medio/medio-alto. Por su parte, McKercher et al. (2008) añade que los aspectos más característicos del perfil sociodemográfico de los turistas gastronómicos es la formación universitaria, el poder adquisitivo medio-alto y una edad que oscila entre los 35 y 45 años. En este sentido, Correia, Kozak y Ferradeira (2009) completan el perfil del gastroturista destacando que las mujeres muestran más interés que los hombres por la gastronomía local, enfatizando de nuevo el nivel educativo superior (formación universitaria). Por su parte, Abdelhamied (2011) también identifica al turista gastronómico con personas cuyo nivel de estudios y de renta es más elevado. Estas conclusiones son reforzadas por otros estudios (Getz, Andersson, Vujicic y Robinson, 2015; López-Guzmán, Hernández-Mogollón y Di Clemente, 2016; Björk y Kauppinen-Räisänen, 2016; Jiménez Beltrán, López-Guzmán y González Santa Cruz, 2016; Levitt et al., 2017) donde se concluye que el turista gastronómico presenta un nivel educativo elevado (normalmente universitario), con un intervalo de edad que abarca desde los 35 a los 45 años, y con un nivel de renta media media y media-alta.

Por tanto, el perfil del turista gastronómico que describe la literatura científica presenta unos aspectos muy concretos y útiles como herramienta de trabajo para el impulso y la consolidación de diferentes destinos turísticos, teniendo en cuenta tanto el interés de esta tipología de viajeros por aprender y por enriquecerse de la cultura del lugar de destino a través de la gastronomía, como el hecho de que estos viajeros tienen un significativo nivel de gasto, lo cual favorece el impacto económico que el destino obtendría si decide desarrollar el turismo gastronómico (Du Rand, Heath y Alberts, 2003).

De acuerdo con la literatura científica, las hipótesis a contrastar serían las siguientes: 
H2: La actitud hacia la gastronomía local está asociada con la edad del turista (McKercher et al., 2008; Correia et al., 2009).

H3: La actitud hacia la gastronomía local está asociada al nivel de formación académica del turista (Getz et al., 2015; López-Guzmán et al., 2016; Jiménez Beltrán e et al. 2016; Levitt et al., 2017).

H4: La actitud hacia la gastronomía local está asociada al nivel de renta del turista (Du Rand et al., 2003).

\subsection{Valoración de la experiencia gastronómica local}

El desarrollo de una propuesta gastronómica atractiva en un determinado destino puede conducir al progreso turístico que, a su vez, logre un notable impacto sobre otros sectores y actividades. Asimismo, este desarrollo permite la diversificación de las actividades turísticas y que, de esta manera, desaparezca la estacionalidad en algunos destinos turísticos. Sin embargo, y para lograr este objetivo, es necesario reforzar una política público-privada apropiada donde se fomenten unos procesos culinarios diferentes en aras de un impulso sostenible del turismo gastronómico, bien mediante unas adecuadas instalaciones, como restaurantes, rutas, infraestructuras, etc., o bien por una promoción del desarrollo de estas actividades culinarias (Ignatov y Smith, 2006). Asimismo, sería interesante combinar las experiencias gastronómicas con otras, como, por ejemplo, el enoturismo o el oleoturismo, convirtiendo así la visita a ese lugar en una experiencia única y diferenciada (Haven-Tang y Jones, 2005).

Sin embargo, y para lograr una experiencia gastronómica memorable, es necesario que la oferta culinaria de ese destino sea al mismo tiempo reconocible e identificable. En este sentido, es importante que exista un número significativo de establecimientos de restauración que hagan posible al visitante contar con una oferta suficiente de cara a una experiencia gastronómica inolvidable (Pérez-Gálvez et al., 2017).

Jiménez Beltrán et al. (2016), en su estudio sobre la gastronomía en la ciudad de Córdoba, destacan la cocina tradicional como un elemento imprescindible tanto para la propia organización de los atractivos de ese destino como para la transferencia de la herencia cultural del lugar hacia el visitante. Por tanto, el desarrollo de procesos culinarios basados en la tradición y en la innovación tiene un carácter esencial, al permitir la preservación de la tradición culinaria y, al mismo tiempo, la innovación con nuevas propuestas gastronómicas (Getz et al., 2015; Kenji, 2016).

De acuerdo con la literatura académica, la hipótesis a contrastar sería la siguiente:

H5: La actitud hacia la gastronomía local determina la experiencia gastronómica del turista (Ignatov y Smith, 2006; Pérez-Gálvez et al., 2017).

\section{METODOLOGÍA}

\subsection{Diseño de la encuesta}

Se desarrolló una metodología cuantitativa, realizando un trabajo de campo sobre una muestra representativa de turistas extranjeros que visitaron la ciudad de Córdoba, con el objetivo de conocer su opinión, su percepción y sus motivaciones en relación con la gastronomía local. La encuesta utilizada en esta investigación está basada en trabajos previos 
diferentes (Kim et al., 2009; Björk y Kauppinen-Räisänen, 2016; López-Guzmán et al., 2017; Pérez-Gálvez et al., 2017) y responde a cuestiones relacionadas con el turismo y la gastronomía. Un borrador de la encuesta se sometió a un proceso de filtro, secuenciado en cuatro fases diferentes: primera, de análisis de los ítems propuestos por un investigador especializado en turismo; segunda, de revisión del cuestionario resultante por varios responsables de la gestión turística de la ciudad de Córdoba; tercera, de revisión del cuestionario por responsables de varios establecimientos culinarios de la ciudad de Córdoba; cuarta, de realización de un pretest a 50 turistas extranjeros. Durante las diferentes fases de depuración de la encuesta se procedió a la corrección de las preguntas que no resultaban fácilmente comprensibles ni a expertos ni a encuestados, obteniendo así la encuesta definitiva que se utilizó en el trabajo de campo.

La versión final de la encuesta buscaba expresar las preguntas con la máxima claridad y el mayor ajuste en las respuestas en aras de los objetivos marcados en la investigación, así como la máxima concreción posible, evitando alargar demasiado la entrevista a los visitantes extranjeros encuestados. El trabajo de campo se realizó en diferentes establecimientos culinarios del centro histórico de la ciudad, y con el requisito de que el turista encuestado llevara un determinado tiempo en el destino y, por tanto, pudiera dar una opinión fundamentada sobre la gastronomía local (Correia et al., 2013; Remoaldo et al., 2014).

La encuesta se divide en tres grandes apartados. El primero de ellos recoge aspectos relacionados con las características del viaje o de la visita como, por ejemplo, el tiempo de permanencia en la ciudad o el tipo de establecimiento en el que pernoctar. El segundo bloque se centra en las materias gastronómicas, como el interés de los encuestados hacia la gastronomía y su motivación a la hora de emprender un viaje, el conocimiento de los platos típicos de la cocina cordobesa, una valoración de los atributos relacionados con los platos degustados o el servicio recibido en los establecimientos culinarios donde fueron consumidos. $Y$ el tercer bloque recoge la información sobre el perfil sociodemográfico de los visitantes, como la edad, el género, el nivel económico, el lugar de procedencia o el nivel de estudios académicos. En la encuesta se utilizaron preguntas con respuestas del tipo sí/no, preguntas con respuestas abiertas y cerradas, y preguntas donde se utilizó una escala de Likert de 5 puntos (1, muy poco importante, y 5, muy importante).

\subsection{Realización del trabajo de campo}

Las encuestas fueron realizadas por un equipo de encuestadores formados para la ocasión y vinculados con la Universidad de Córdoba. Los cuestionarios se pasaron en dos idiomas, español e inglés, y cada encuestado elegía el idioma para la realización del mismo.

La muestra objeto de estudio estuvo representada por 753 turistas, con una tasa de rechazo del 1\%. En ningún caso la duración de la encuesta superó los 10 minutos.

El desarrollo del trabajo de campo se efectuó entre los meses de marzo y septiembre de 2018. Los cuestionarios fueron cumplimentados en diversos establecimientos culinarios de la zona turística de la ciudad de Córdoba, en días y horarios distintos, tratando así de recoger el abanico más amplio posible de personas y situaciones. Se utilizó un muestreo técnico no probabilístico, comúnmente utilizado en este tipo de investigaciones donde los encuestados están disponibles para ser encuestados en un espacio y en un tiempo determinado (Finn, Elliott-White y Walton, 2000). Por tanto, se utilizó un muestreo de conveniencia y no se realizó 
estratificación alguna por género, edad, formación, nacionalidad ni otra variable por la falta de estudios previos que la avalasen.

\subsection{Muestreo y error muestral}

El marco concreto de esta investigación es el turista extranjero que visita la ciudad de Córdoba, con independencia de si pernocta o no, o de si visita o no otros lugares cercanos. En relación con el número de turistas extranjeros que viajan a la ciudad, se ha elegido como dato de referencia el número de turistas extranjeros que se alojaron en establecimientos hoteleros de la ciudad en 2017. El número de turistas extranjeros en 2017 ascendió, según la Encuesta de Ocupación Hotelera del Instituto Nacional de Estadística -INE- (2019), a 482.334. Por tanto, partiendo de este número de visitantes extranjeros, y con carácter orientativo, si en la investigación se hubiese utilizado un muestreo aleatorio simple, el error muestral para un nivel de confianza del $95 \%$ sería del $\pm 3,67 \%$.

\subsection{Análisis de los datos}

La tabulación y el análisis estadístico de los datos se han realizado utilizando el programa informático SPSS v. 24. Se han utilizado estadísticos para valorar la fiabilidad y la validez de las respuestas al cuestionario aplicado (alpha de Cronbach). Asimismo, se ha aplicado la técnica multivariante de agrupación de casos (conglomerados K-medias) con el objeto de analizar la similitud o el parecido existente entre los encuestados, tomando como referencias variables indicativas del mayor o del menor interés hacia la gastronomía como variable de interés a la hora de viajar. Asimismo, se ha empleado la técnica del análisis discriminante para validar la agrupación de casos obtenidos a través del análisis de conglomerados. A partir de los grupos o segmentos obtenidos se han aplicado estadísticos y medidas de asociación que proporcionan la información necesaria para estudiar las posibles pautas de asociación existentes entre las diferentes variables a partir de una tabla de contingencias bidimensional. Por otro lado, se han empleado procedimientos estadísticos no paramétricos ( $\mathrm{H}$ de Krustal-Wallis y $U$ de Mann-Whitney) con el objetivo de analizar las diferencias significativas existentes entre los diferentes grupos de la muestra obtenida.

\section{RESULTADOS DE LA INVESTIGACIÓN}

\subsection{Segmentación de los gastroturistas extranjeros}

Con el objetivo de analizar y de caracterizar el interés por la gastronomía de los turistas extranjeros que visitaron la ciudad de Córdoba, se solicitó a los encuestados que evaluasen la importancia de la gastronomía en sus viajes a partir de tres preguntas diferentes. Estas preguntas están basadas en el modelo planteado por Björk y Kauppinen-Räisänen (2016) para segmentar a los turistas gastronómicos. El coeficiente alpha de Cronbach de la escala final alcanza un valor de 0,809 , indicativo de una consistencia interna meritoria entre los elementos de la escala. El nivel crítico (p), asociado al estadístico $\chi^{2}$ de Friedman $(57,744)$ del análisis para contrastar la hipótesis nula de que todos los elementos de la escala tienen la misma media, es menor que 0,05 . Por tanto, se rechaza la hipótesis de que las medias de los elementos sean iguales.

La literatura científica ha confiado en varios enfoques para la segmentación de los consumidores. Una de las técnicas más comunes en la investigación de turismo es el análisis factor-clúster (Park y Yoon, 2009; Prayag, 2010). No obstante, este enfoque ha sido objeto de 
crítica por deficiencias observadas, como la pérdida de información original, la interpretación abstracta o las posibles suposiciones erróneas (Dolnicar, 2008; Dolnicar, Kaiser, Lazarevski y Leisch, 2012; Prayag y Hosany, 2014). Esta investigación emplea el enfoque de segmentación recomendado por Dolnicar (2008), que implica la agrupación directa de las puntuaciones originales. El uso de éstas ofrece una segmentación más precisa o detallada debido a su capacidad para retener un mayor grado de los datos originales (Sheppard, 1996; Dolnicar, 2002; Prayag y Hosany, 2014).

La literatura científica recomienda el uso de un método de agrupamiento jerárquico seguido de un método no jerárquico (Hair, Black, Babin y Anderson, 2010). Por lo tanto, dos algoritmos jerárquicos, a saber, el enlace completo y el método de Ward, fueron aplicados inicialmente en esta investigación, utilizando distancias euclidianas cuadradas para identificar posibles agrupaciones en los datos obtenidos. Ambas técnicas prevalecen en la literatura científica turística basada en la segmentación del mercado (Dolnicar, 2002). El objetivo de esta investigación es identificar grupos o segmentos de turistas que sean muy similares en cuanto a su interés por la gastronomía local a la hora de viajar. Un examen de los cronogramas de aglomeración resultantes y de los dendrogramas sugirió tres o cuatro soluciones de clústeres. Un examen más detallado de la asignación grupal y del tamaño del grupo, y el posterior análisis utilizando un algoritmo de agrupación K-means no jerárquico, confirmó que la solución más adecuada era la segmentación de los turistas extranjeros en tres grupos, segmentos o clústeres.

Siguiendo el modelo de Björk y Kauppinen-Räisänen (2016), cada uno de estos segmentos ha sido denominado como: turista superviviente (survivors), disfrutadores (enjoyers) y experimentadores (experiencers). Los resultados se recogen en la tabla 1.

Tabla 1. Segmentación de los turistas en base a su interés por la gastronomía

\begin{tabular}{|c|c|c|c|c|c|}
\hline \multirow{2}{*}{ Actitud hacia la gastronomía } & \multicolumn{3}{|c|}{ Clústeres } & \multicolumn{2}{|c|}{ H-Kruskal Wallis } \\
\hline & $\begin{array}{l}\text { Supervi- } \\
\text { vientes }\end{array}$ & $\begin{array}{l}\text { Disfruta- } \\
\text { dores }\end{array}$ & $\begin{array}{l}\text { Experimen- } \\
\text { tadores }\end{array}$ & $\chi^{2}$ & Sig. \\
\hline $\begin{array}{l}\text { ¿Qué supone la gastronomía en su } \\
\text { motivación para viajar? }\end{array}$ & $2,02^{(*)}$ & $3,11^{(*)}$ & $4,38^{(*)}$ & 430,788 & $<0,000$ \\
\hline $\begin{array}{l}\text { ¿Busca experiencias gastronómicas } \\
\text { cuando elige un destino para visitarlo? }\end{array}$ & $1,85^{(*)}$ & $3,28^{(*)}$ & $\left.4,35^{*}\right)$ & 416,440 & $<0,000$ \\
\hline $\begin{array}{l}\text { ¿La experiencia gastronómica es } \\
\text { importante en la satisfacción de su } \\
\text { viaje? }\end{array}$ & $2,35^{(*)}$ & $\left.3,57^{*}\right)$ & $4,45^{(*)}$ & 381,579 & $<0,000$ \\
\hline $\begin{array}{l}\text { (*) Los valores en negrita presentan dife } \\
\text { probar las diferencias significativas entre }\end{array}$ & $\begin{array}{l}\text { encias signi } \\
\text { los diferent }\end{array}$ & $\begin{array}{l}\text { ativas en do } \\
\text { segmentos }\end{array}$ & $\begin{array}{l}\text { de los tres grup } \\
\text { e aplicó la prueb }\end{array}$ & $\begin{array}{l}\text { de segn } \\
\text { U-Mann }\end{array}$ & $\begin{array}{l}\text { ntos. Para } \\
\text { hitney. }\end{array}$ \\
\hline
\end{tabular}

Elaboración propia

A partir de la tabla 1 puede observarse que el primero de los segmentos está integrado por el $14,6 \%$ de los turistas extranjeros encuestados, siendo el grupo que anota unos significativos bajos registros en los tres ítems. A este clúster se le denomina "turistas 
supervivientes", integrado por turistas que muestran un bajo interés por la gastronomía. El segundo grupo representa el $38,1 \%$ de la muestra y se caracteriza por anotar puntuaciones intermedias en los tres ítems. Este clúster, al que se le denomina "turistas disfrutadores", agrupa a los turistas con un relativo interés por la gastronomía. El tercero de los grupos se caracteriza por anotar elevadas puntuaciones en los tres ítems y representa el 47,3\% de los encuestados. Este porcentaje es un poco superior a lo detectado por la literatura previa (McKercher et al., 2008; Thompson y Prideaux, 2009; Björk y Kauppinen-Räisänen, 2016) y se debe al lugar donde se realizaron las encuestas. No obstante, sí confirma los resultados obtenidos por la literatura científica previa en el sentido de que existen dos grupos de turistas, uno nada interesados en la gastronomía local, y otro grupo muy interesado en la gastronomía local. A este clúster, que agrupa a los turistas con un mayor interés por la gastronomía, se le ha denominado "turistas experimentadores". El estadístico H de Kruskal Wallis (1952) permite contrastar que las medias comparadas no son iguales entre los diferentes clústeres, pero no permite precisar dónde se encuentran las diferencias detectadas. Para saber qué media difiere de la otra, se ha recurrido al estadístico U de Mann-Whitney (1947).

En la línea de otras investigaciones previas (Thompson y Prideaux, 2009; Björk y Kauppinen-Räisänen, 2016; López-Guzmán et al., 2017; Pérez-Gálvez et al., 2017), los resultados permiten contrastar una de las hipótesis de trabajo planteadas: los turistas muestran actitudes diferentes hacia la gastronomía como variable de interés en la elección del destino (H1).Los tres grupos obtenidos están reflejados en la literatura científica, donde las investigaciones realizadas segmentando grupos de turistas señalan, al menos, uno de los grupos como muy interesado en la gastronomía y otro como muy poco interesado. Así, y en relación con lo que en esta investigación se denomina "turistas experimentadores", es un grupo que ha sido identificado en investigaciones anteriores. Hjalaberg (2004) lo denomina experimental gastronomy tourists y McKercher et al. (2008) lo identifica definite culinary tourist. En sentido opuesto, se da con el grupo denominado en esta investigación "turistas supervivientes". Hjalaberg (2004) caracteriza a uno de los grupos como recreational, McKercher et al. (2008) como non culinary tourist o Thompson y Prideaux (2009) como not interested.

\subsection{Perfil sociodemográfico del turista extranjero y características del viaje}

De las 710 personas entrevistadas, el 38,5\% fueron hombres y el 61,5\% restante mujeres, no existiendo diferencias importantes en la toma de datos a lo largo de los diferentes días. Los visitantes encuestados son, en términos generales, jóvenes. La tabla 2 refleja cómo más del $70 \%$ de la muestra tienen menos de 40 años. A diferencia de otras investigaciones (Tse y Crotts, 2005; Pérez-Gálvez et al., 2017), no se detectan diferencias en función de los segmentos de turistas identificados (estadístico $\mathrm{H}$ de Kruskal Wallis $=0,854 ; \mathrm{p} \mathrm{0,653)}$ ). Este resultado no permite contrastar otra de las hipótesis de investigación planteadas: La actitud hacia la gastronomía local está asociada con la edad del turista ( $\mathrm{H} 2)$.

El nivel de formación académica de los encuestados es elevado, tal y como refleja la tabla 2. Así, un $72,0 \%$ de los encuestados declaran contar con un grado o posgrado universitario. Al analizar el nivel de estudios en función de la edad no se observan diferencias (coeficiente gamma $=0,081 ; p=0,127$ ). Asimismo, y considerando la variable edad, no se detectan diferencias por clústeres gastronómicos (estadístico $\mathrm{H}$ de Kruskal Wallis = 5,533; $\mathrm{p}=$ $0,063)$. Estos resultados no avalarían la hipótesis $(\mathrm{H} 3)$ relativa a la asociación entre la actitud 
hacia la gastronomía local y la formación académica del turista (Kivela y Crotss, 2005; Ignatov y Smith, 2006; McKercher et al., 2008; Jiménez Beltrán et al., 2016; Levitt et al., 2017).

Tabla 2. Perfil sociodemográfico del turista extranjero en la ciudad de Córdoba

\begin{tabular}{|c|c|c|c|c|c|}
\hline \multirow[b]{2}{*}{ Variables } & \multirow[b]{2}{*}{ Categorías } & \multicolumn{3}{|c|}{ Clústeres } & \multirow[b]{2}{*}{ Total } \\
\hline & & $\begin{array}{l}\text { Supervi- } \\
\text { vientes }\end{array}$ & $\begin{array}{l}\text { Disfruta- } \\
\text { dores }\end{array}$ & $\begin{array}{l}\text { Experimen- } \\
\text { tadores }\end{array}$ & \\
\hline $\begin{array}{l}\text { Género } \\
(N=707)\end{array}$ & $\begin{array}{l}\text { Hombre } \\
\text { Mujer }\end{array}$ & $\begin{array}{l}35,6 \% \\
64,4 \%\end{array}$ & $\begin{array}{l}40,1 \% \\
59,9 \%\end{array}$ & $\begin{array}{l}38,0 \% \\
62,0 \%\end{array}$ & $\begin{array}{l}38,5 \% \\
61,5 \%\end{array}$ \\
\hline $\begin{array}{l}\text { Edad } \\
(N=709)\end{array}$ & $\begin{array}{l}\text { Menos de } 30 \text { años } \\
30-39 \text { años } \\
40-49 \text { años } \\
50-59 \text { años } \\
60 \text { años o más }\end{array}$ & $\begin{array}{r}51,0 \% \\
16,3 \% \\
16,3 \% \\
9,6 \% \\
6,7 \%\end{array}$ & $\begin{array}{r}58,1 \% \\
13,7 \% \\
10,0 \% \\
8,5 \% \\
9,6 \%\end{array}$ & $\begin{array}{r}56,1 \% \\
15,2 \% \\
12,2 \% \\
9,6 \% \\
6,9 \%\end{array}$ & $\begin{array}{r}56,1 \% \\
14,8 \% \\
12,0 \% \\
9,2 \% \\
7,9 \%\end{array}$ \\
\hline $\begin{array}{l}\text { Nivel de } \\
\text { formación } \\
(N=706)\end{array}$ & $\begin{array}{l}\text { Educación primaria } \\
\text { Educación secundaria } \\
\text { Educación universitaria } \\
\text { Master/PhD }\end{array}$ & $\begin{array}{r}1,0 \% \\
31,7 \% \\
42,6 \% \\
24,8 \%\end{array}$ & $\begin{array}{r}1,5 \% \\
21,6 \% \\
46,1 \% \\
30,9 \%\end{array}$ & $\begin{array}{r}4,5 \% \\
26,2 \% \\
43,2 \% \\
26,2 \%\end{array}$ & $\begin{array}{r}2,8 \% \\
25,2 \% \\
44,2 \% \\
27,8 \%\end{array}$ \\
\hline $\begin{array}{l}\text { Ocupación } \\
\text { (N = 702) }\end{array}$ & $\begin{array}{l}\text { Prof. independiente } \\
\text { Empleado publico } \\
\text { Trab. empresa privada } \\
\text { Autónomo } \\
\text { Estudiante } \\
\text { Desempleado } \\
\text { Jubilado } \\
\text { Labores del hogar }\end{array}$ & $\begin{array}{r}17,3 \% \\
13,5 \% \\
22,1 \% \\
3,8 \% \\
36,5 \% \\
----- \\
6,7 \% \\
-----\end{array}$ & $\begin{array}{r}12,2 \% \\
16,0 \% \\
17,9 \% \\
3,8 \% \\
37,6 \% \\
1,1 \% \\
8,4 \% \\
3,0 \%\end{array}$ & $\begin{array}{r}10,1 \% \\
13,1 \% \\
29,6 \% \\
2,4 \% \\
34,9 \% \\
2,4 \% \\
5,4 \% \\
2,1 \%\end{array}$ & $\begin{array}{r}12,0 \% \\
14,2 \% \\
24,1 \% \\
3,1 \% \\
36,2 \% \\
1,6 \% \\
6,7 \% \\
2,1 \%\end{array}$ \\
\hline $\begin{array}{l}\text { Lugar de } \\
\text { procedencia } \\
(\mathrm{N}=710)\end{array}$ & $\begin{array}{l}\text { Europa } \\
\text { América } \\
\text { Resto del Mundo }\end{array}$ & $\begin{array}{r}85,6 \% \\
7,7 \% \\
6,7 \%\end{array}$ & $\begin{array}{r}81,5 \% \\
15,9 \% \\
2,6 \%\end{array}$ & $\begin{array}{r}75,3 \% \\
19,3 \% \\
5,4 \%\end{array}$ & $\begin{array}{r}79,2 \% \\
16,3 \% \\
4,5 \%\end{array}$ \\
\hline
\end{tabular}

Elaboración propia

En cuanto a la categoría profesional de los turistas encuestados, prevalecen los estudiantes, los trabajadores asalariados y los empleados públicos. Respecto del lugar de procedencia de los visitantes, el turismo europeo representa un 79,2\% de los turistas extranjeros que llega Córdoba, seguido del americano con un 16,3\%. Por países, destacan los visitantes procedentes de Reino Unido $(15,1 \%)$, Francia $(14,1 \%)$, Italia $(10,8 \%)$ y Estados Unidos (8,9\%). 
El análisis del nivel mensual de renta familiar revela que un $13,7 \%$ de los visitantes encuestados declaran tener un ingreso inferior a los 1.000 euros mensuales frente al $49,4 \%$ que afirman ganar más de 2.500 euros (tabla 3), lo que pone de manifiesto que los turistas extranjeros que visitan la ciudad de Córdoba cuentan con una capacidad adquisitiva alta. El nivel medio de renta declarado por los turistas englobados en el segmento denominado "turistas experimentadores" es algo superior al resto de segmentos, sin que lleguen a existir diferencias significativas (estadístico $\mathrm{H}$ de Kruskal Wallis $=4,415 ; \mathrm{p}=0,110$ ). Por tanto, estos resultados no permiten avalar la hipótesis $(\mathrm{H} 4)$ relativa a la asociación entre la actitud hacia la gastronomía local y el nivel de ingresos del turista (Fields 2002; Hall et al., 2003; Du Rand et al., 2003).

En cuanto al grado de repetición del viaje destaca un alto índice de repetición de la visita a Córdoba (81,3\%), siendo más elevado cuanto mayor es el interés de los turistas en la gastronomía local. Así, el segmento denominado "turistas experimentadores" presenta un índice de repetición del 86,0\%. En este sentido, se aprecian diferencias significativas respecto al resto de segmentos turísticos (estadístico H de Kruskal Wallis =12,653; $p=0,002$ ).

Entre los visitantes entrevistados, el 36,0\% declara no pernoctar en la ciudad y el 53,3\% hacerlo al menos dos noches. En cuanto al tipo de alojamiento utilizado, el apartamento turístico es el más solicitado, seguido del hotel de cuatro o cinco estrellas, y del hotel de dos o tres estrellas (tabla 3 ).

Tabla 3. Características del viaje.

\begin{tabular}{|c|c|c|c|c|c|}
\hline \multirow{2}{*}{ Variables } & \multirow{2}{*}{ Categorías } & \multicolumn{3}{|c|}{ Segmentos } & \multirow{2}{*}{ Total } \\
\hline & & $\begin{array}{l}\text { Supervi- } \\
\text { vientes }\end{array}$ & $\begin{array}{l}\text { Disfruta- } \\
\text { dores }\end{array}$ & $\begin{array}{l}\text { Experimen- } \\
\text { tadores }\end{array}$ & \\
\hline $\begin{array}{l}\text { Repetición del } \\
\text { viaje } \\
(\mathrm{N}=710)\end{array}$ & $\begin{array}{l}\text { Sí } \\
\text { No }\end{array}$ & $\begin{array}{l}71,2 \% \\
28,8 \%\end{array}$ & $\begin{array}{l}79,3 \% \\
20,7 \%\end{array}$ & $\begin{array}{l}86,0 \% \\
14,0 \%\end{array}$ & $\begin{array}{l}81,3 \% \\
18,7 \%\end{array}$ \\
\hline $\begin{array}{l}\text { Renta } \\
=567)\end{array}$ & $\begin{array}{l}\text { Menos de } 700 € \\
\text { De } 700 € \text { a } 999 € \\
\text { De } 1.000 € \text { a } 1.499 € \\
\text { De } 1.500 € \text { a } 2.499 € \\
\text { De } 2.500 € \text { a } 3.500 € \\
\text { Más de } 3.500 €\end{array}$ & $\begin{array}{r}8,7 \% \\
2,9 \% \\
21,7 \% \\
20,3 \% \\
14,5 \% \\
31,9 \%\end{array}$ & $\begin{array}{r}9,1 \% \\
6,4 \% \\
14,6 \% \\
23,3 \% \\
19,2 \% \\
27,4 \%\end{array}$ & \begin{tabular}{r|}
$6,8 \%$ \\
$6,1 \%$ \\
$11,5 \%$ \\
$23,3 \%$ \\
$14,7 \%$ \\
$37,6 \%$
\end{tabular} & $\begin{array}{r}7,9 \% \\
5,8 \% \\
13,9 \% \\
22,9 \% \\
16,4 \% \\
33,0 \%\end{array}$ \\
\hline $\begin{array}{l}\text { Estancia } \\
(\mathrm{N}=702)\end{array}$ & $\begin{array}{l}\text { No pernocto } \\
\text { Una noche } \\
\text { Dos noches } \\
\text { Más de dos noches }\end{array}$ & $\begin{array}{l}35,9 \% \\
12,6 \% \\
17,5 \% \\
34,0 \%\end{array}$ & $\begin{array}{l}34,3 \% \\
10,9 \% \\
23,0 \% \\
31,7 \%\end{array}$ & $\begin{array}{r}37,4 \% \\
9,9 \% \\
30,5 \% \\
22,2 \%\end{array}$ & $\begin{array}{l}36,0 \% \\
10,7 \% \\
25,8 \% \\
27,5 \%\end{array}$ \\
\hline $\begin{array}{l}\text { Pernoctación } \\
(\mathrm{N}=386)\end{array}$ & $\begin{array}{l}\text { Hoteles } 4-5 \text { estrellas } \\
\text { Hoteles } 2-3 \text { estrellas } \\
\text { Hotel una Estrella/hostel } \\
\text { Casa de familiares o amigos } \\
\text { Apartamento turístico }\end{array}$ & $\begin{array}{r}20,4 \% \\
18,5 \% \\
24,1 \% \\
7,4 \% \\
29,6 \%\end{array}$ & $\begin{array}{r}18,1 \% \\
20,1 \% \\
20,1 \% \\
5,6 \% \\
36,1 \%\end{array}$ & $\begin{array}{l}31,9 \% \\
20,2 \% \\
11,7 \% \\
10,6 \% \\
25,5 \%\end{array}$ & $\begin{array}{r}25,1 \% \\
19,9 \% \\
16,6 \% \\
8,3 \% \\
30,1 \%\end{array}$ \\
\hline
\end{tabular}

Elaboración propia 


\subsection{Valoración experiencia gastronomía local}

La gastronomía local cordobesa es conocida para el turista extranjero que visita la ciudad. Los turistas encuestados fueron preguntados sobre su conocimiento acerca de los tres platos típicos de la cocina cordobesa (salmorejo, rabo de toro y flamenquín). Un 38,0\% de los encuestados conocían al menos dos de estos platos y un 14,1\% conocían los tres, siendo el salmorejo el plato más conocido por los turistas, mientras que el rabo de toro es el menos conocido. Asimismo, se les solicitó una valoración sobre una relación de los atributos de la gastronomía local con el fin de detectar fortalezas y puntos de mejora -tabla 4-. La estimación de algunos atributos no es tan alta como el grado de satisfacción general ya que son aspectos muy concretos en cuya valoración intervienen factores tanto de carácter personal como particular. Los atributos más valorados son la gastronomía local, el ambiente de los establecimientos, y el servicio y la hospitalidad. La menor valoración afecta a la innovación, a los nuevos sabores en los platos y a la variedad de las especialidades gastronómicas locales.

Tabla 4. Valoración de los atributos de la gastronomía en Córdoba

\begin{tabular}{|l|r|r|}
\hline Atributos & Media & Ranking \\
\hline Calidad de los platos & 3,74 & 4 \\
\hline Variedad de platos & 3,55 & 7 \\
\hline Precios & 3,65 & 5 \\
\hline Instalaciones & 3,61 & 6 \\
\hline Ambiente de los establecimientos & 3,91 & 2 \\
\hline Innovación y nuevos sabores en los platos & 3,51 & 8 \\
\hline Servicio y hospitalidad & 3,83 & 3 \\
\hline Gastronomía tradicional & 3,92 & 1 \\
\hline & & 0,899 \\
\hline
\end{tabular}

Elaboración propia

El análisis por segmentos muestra una percepción de la gastronomía cordobesa por parte de los turistas extranjeros significativamente distinta (Kim et al., 2009; Kim et al., 2013; Timothy y Ron, 2013; López-Guzmán et al., 2017; Pérez-Gálvez et al., 2017) -tabla 5-. Así, todos los atributos gastronómicos son más valorados por el segmento de turistas que presentan un mayor interés por la gastronomía (H5). 
Tabla 5. Caracterización por segmentos de la valoración de los atributos de la gastronomía en Córdoba

\begin{tabular}{|c|c|c|c|c|c|}
\hline \multirow{2}{*}{$\begin{array}{l}\text { Atributos de la } \\
\text { gastronomía }\end{array}$} & \multicolumn{3}{|c|}{ Segmentos } & \multicolumn{2}{|c|}{$\begin{array}{l}\text { H-Kruskal } \\
\text { Wallis }\end{array}$} \\
\hline & Supervivientes & Disfrutadores & Experimentadores & $x^{2}$ & Sig. \\
\hline $\begin{array}{l}\text { Calidad de los } \\
\text { platos }\end{array}$ & $3,10^{(*)}$ & $3,64^{(*)}$ & $4,02^{(*)}$ & 86,734 & $<0,000$ \\
\hline $\begin{array}{l}\text { Variedad de } \\
\text { platos }\end{array}$ & $\left.3,09^{*}\right)$ & $3,44^{(*)}$ & $3,79^{(*)}$ & 45,473 & $<0,000$ \\
\hline Precios & $3,14^{(*)}$ & $3,56^{(*)}$ & $3,88^{(*)}$ & 39,681 & $<0,000$ \\
\hline Instalaciones & $3,12^{(*)}$ & $3,50^{(*)}$ & $3,85^{(*)}$ & 49,617 & $<0,000$ \\
\hline $\begin{array}{l}\text { Ambiente de los } \\
\text { establecimientos }\end{array}$ & $3,48^{(*)}$ & $3,85^{(*)}$ & $4,07^{(*)}$ & 27,227 & $<0,000$ \\
\hline $\begin{array}{l}\text { Innovación y } \\
\text { nuevos sabores }\end{array}$ & $3,05^{(*)}$ & $3,38^{(*)}$ & $3,75^{(*)}$ & 41,685 & $<0,000$ \\
\hline $\begin{array}{l}\text { Servicio y } \\
\text { hospitalidad }\end{array}$ & $3,25^{(*)}$ & $3,81^{(*)}$ & $4,02^{(*)}$ & 38,208 & $<0,000$ \\
\hline $\begin{array}{l}\text { Gastronomía } \\
\text { tradicional }\end{array}$ & $3,30^{(*)}$ & $3,80^{(*)}$ & $4,20^{(*)}$ & 58,437 & $<0,000$ \\
\hline
\end{tabular}

Elaboración propia

\section{CONCLUSIONES}

En la actualidad, el turismo gastronómico constituye una de las opciones para incrementar o consolidar determinados destinos turísticos, debido a la importancia, cada vez mayor, que los viajeros otorgan al conocimiento de todo lo relacionado con la cultura gastronómica de los lugares que visitan. De hecho, el ir a un restaurante determinado o simplemente conocer mejor la cocina de una zona geográfica concreta son considerados por determinados viajeros como motivación principal de su viaje. En esta investigación se ha analizado la relación existente entre el turismo extranjero y la gastronomía en un destino inscrito como Patrimonio de la Humanidad, la ciudad de Córdoba. Los turistas que visitan un destino cultural, además de profundizar en el conocimiento de su patrimonio, también quieren disfrutar de experiencias sensoriales. En este sentido, la gastronomía y su relación con el turismo se ha convertido en un aspecto clave del análisis de los destinos turísticos, especialmente de aquellos relacionados con la cultura y con el patrimonio. La relación entre gastronomía y cultura no sorprende si se tiene en cuenta que casi un $70 \%$ de los turistas encuestados en esta investigación tienen una formación académica universitaria.

La gastronomía local adquiere un título de atracción turística y constituye un objetivo notable cuando se visita un destino turístico. En este sentido, esta investigación concluye que los turistas extranjeros manifiestan actitudes diferentes hacia la gastronomía local como variable de desarrollo turístico. Atendiendo al interés declarado hacia la gastronomía en los 
viajes, se obtiene una evidencia empírica sobre la presencia de tres tipologías de turistas, denominadas, siguiendo el modelo de Björk y Kauppinen-Räisänen (2016), turistas supervivientes, turistas disfrutadores y turistas experimentadores. Esta segmentación se considera validada y de utilidad para agrupar a los visitantes desde un punto de vista gastronómico. De los tres clústeres identificados, la gastronomía desempeña un papel relevante entre los turistas con un alto interés gastronómico denominados "turistas experimentadores".

El perfil sociodemográfico del turista extranjero que visita la ciudad de Córdoba es un turista con un elevado nivel de formación, procede fundamentalmente del resto de países europeos y presenta, por término medio, un nivel de renta medio y medio-alto, contando los turistas englobados en el segmento denominado "turistas experimentadores" con una mayor renta.

Los visitantes expresan una alta satisfacción con su experiencia culinaria, siendo la valoración significativa distinta atendiendo al interés declarado hacia la gastronomía. De igual manera, el mayor interés se traduce en percepciones significativamente distintas en relación con los atributos de la cocina local, siendo valoradas en mayor medida la gastronomía tradicional, los precios y la calidad de la comida. De acuerdo con las opiniones de los turistas extranjeros encuestados, entre los atributos mejor posicionados destacan la gastronomía local, el ambiente de los establecimientos, y el servicio y la hospitalidad. No obstante, serían objeto de estudio las posibilidades de mejora en lo concerniente a la innovación, a los nuevos sabores en los platos o a la variedad de las especialidades gastronómicas locales.

Esta investigación no sólo proporciona implicaciones teóricas sino también prácticas. Su principal aplicación práctica es contribuir a entender las características y elementos diferenciadores de los diferentes grupos de turistas extranjeros identificados y la valoración que realizan de la gastronomía local con la finalidad de concebir productos turísticos y culturales que satisfagan mejor sus necesidades $y$, al mismo tiempo, sean compatibles con la gestión sostenible de la gastronomía local. En este sentido, y con el fin de que la gastronomía local se convierta en una herramienta más de competitividad turística se hace necesario establecer medidas que favorezcan la innovación en los platos.

Como limitaciones de esta investigación cabe señalar el periodo temporal de realización del trabajo de campo, considerando necesaria una ampliación del estudio durante todos los meses del año para evitar posibles sesgos temporales y el lugar de realización de las encuestas. Por otra parte, un análisis completo del sector turístico de Córdoba exigiría realizar una investigación paralela de las empresas de la oferta turística. Por esta razón, como futura línea de investigación, se recomienda realizar una investigación profunda del lado de la oferta gastronómica enfocado al turista en la ciudad de Córdoba.

\section{REFERENCIAS BIBLIOGRÁFICAS}

Abdelhamied, H. H. S. (2011). Customers' perceptions of floating restaurants in Egypt. Anatolia, 22(1), 1-15.

Alonso, A. D., Fraser, R. A. y Cohen, D.A. (2007). Investigating differences between domestic and international winery visitors in New Zealand. International Journal of Wine Business Research, 19 (2), 114-126. 
Anderson, T. D., Musberg, L. y Therkelsen, A. (2017). Food and tourism synergies: perspectives on consumption, production and destination development. Scandinavian Journal of Hospitality and Tourism, 17(2), 1-8.

Babolian hendijani, R. (2016). Effect of food experience on tourist satisfaction: the case of Indonesia. International Journal of Culture, Tourism and Hospitality Research, 10(3), 272-282.

Baker, D. A. y Crompton, J. L. (2000). Quality, satisfaction and behavioral intentions. Annals of Tourism Research, 27(3), 785-804.

Basil, M. D. y Basil, D. Z. (2009). Reflections of ultra-fine dining experiences. En Lindgreen, A., Vanhammed, J. y Bervelan, M. B. (eds.). Memorable customer experiences: a research anthology (135-147). Surrey: Gower Publishing Company.

Björk, P. y Kauppinen-Räisänen, H. (2016). Exploring the multi-dimensionality of travellers' culinary-gastronomic experiences. Current Issues in Tourism, 19(12), 1260-1280.

Björk, P. y Kauppinen-Räisänen, H. (2017). A destination's gastronomy as a means for holiday well-being. British Food Journal, 119(7), 1578-1591.

Boniface, P. (2003). Tasting tourism: travelling for food and drink. Aldershot: Ashgate Publishing Limited.

Carrien, A., Duthra, I. B., Texeira, J. C., Gontijo, B. M. y Tijoux, M. E. (2012). Metaforseando los mercados centrales. Estudios y Perspectivas en Turismo, 12, 88-107.

Charters, S. y Ali-Knight, J. (2002). Who is the wine tourist? Tourism management, 23(3), 311319.

Chen, C. F. y Chen. F. S. (2010). Experience quality. perceive value. satisfaction. and behavioral intentions for heritage tourists. Tourism Management, 31(1). 29-35.

Chen, C.F. y Tsai. D. (2007). How destination image and evaluative factors affect behavioural intentions? Tourism Management, 28, 1115-1122.

Cohen, E. y Avieli, N. (2004). Food in tourism: attraction and impediment. Annals of Tourism Research, 31(4), 755-778.

Correia, A., Kozak, M. y Ferradeira, J. (2013). From tourist motivations to tourist satisfaction. International Journal of Culture, Tourism and Hospitality Research, 7(4), 411-424.

Cracolici, M. F., Nijkamp, P. y Rietveld, P. (2008). Assessment of tourism competitiveness by analysing destination efficiency. Tourism Economics, 14(2), 325-342.

Crespi-Vallbona, M. y Domínguez Pérez, M. (2016). Los mercados de abastos y las ciudades turísticas. Pasos, Revista de Turismo y Patrimonio Cultural, 14(2), 401-416.

Croce, E. y Perri, G. (2010). Food and wine tourism. Oxford: Cabi.

De Jong, A., Palladino, M., Garrido Puig, R., Romeo, G., Fava, N., Cafiero, C., Skoglund, W., Varley, P., Marciano, C., Laven, D. y Sjölander-Lindqvist, A. (2018). Gastronomy tourism: An interdisciplinary literatura review of research areas, disciplines, and dynamics. Journal of Gastronomy and Tourism, 3, 131-146. 
Desmet, P.M.A. y Schifferstein, H.N.J. (2008). Sources of positive and negative emotions in food experience. Appetite, 50, 290-301.

Dimitrovski, D. y Crespi-Vallbona, M. (2017). Role of food neophilia in food market tourists' motivational construct: The case of La Boqueria in Barcelona, Spain. Journal of Travel \& Tourism Marketing, 34(4), 475-487.

Dimitrovski y Crespi-Vallbona (2018). Urban food markets in the context of a tourist attractionLa Boqueria market in Barcelona, Spain. Tourism Geographies, 20(3), 397-417.

Dixit, S. K. (2019). The Routledge handbook of gastronomic tourism. Londres: Routledge International.

Dolnicar, S. (2002). A review of data-driven market segmentation in tourism. Journal of Travel \& Tourism Marketing, 12(1), pp. 1-22.

Dolnicar, S. (2008). Market segmentation in tourism. En Woodside, A. and Martin, D. (Eds.), Tourism management, analysis, behavior and strategy (pp. 129-150). Cambridge: CABI.

Dolnicar, S., Kaiser, S., Lazarevski, K. y Leisch, F. (2012). Biclustering: overcoming data dimensionality problems in market segmentation. Journal of Travel Research, 51(1), 41-49.

Du Rand, G. E., Heath, E. y Alberts, N. (2003). The role of local and regional food in destination marketing: A South African situation analysis. Journal of Travel and Tourism Marketing, 14(3/4), 37-52.

Ellis, A. Park, E., Kim, S. y Yeoman, I. (2018). What is food tourism? Tourism Management, 68, 250-263.

Fields, K. (2002). Demand for the gastronomy tourism product. Motivational factors. En Hjalager, A. M. y Richards, G. (Eds.). Tourism and Gastronomy (pp. 36-50). London: Routledge.

Finn M., Elliott-White M. yWalton M. (2000). Tourism and leisure research methods: Data collection, analysis and interpretation. Harlow: Pearson Education.

Fox, R. (2007). Reinventing the gastronomic identity of Croatian tourist destinations. International Journal of Hospitality Management, 26, 546-559.

Gagic, S. M., Tesanovic, D. V., Ivkox-Dzigurski, A. C., Drago Pirau, T. y Jovicic, A. D. (2013). Motives and attitudes of food and drink festival visitors: A case study of Serbia. Journal of Food, Agriculture and Environment, 11(1), 1055-1059.

Getz, D., Robinson, R., Anderson, T. y Vujicic, S. (2014). Foodies and food tourism. Oxford: Goodfellow.

Getz, D. (2008). Even tourism: definition, evolution, and research. Tourism Management, 29, 403-428.

Ghatak, I. y Chatterjee, S. (2018). Urban street vending practices: an investigation of ethnic food safety knowledge, attitudes, and risks among untrained Chinese vendors in Chinatown, Kolkata. Journal of Ethnic Foods, 5, 272-285.

Hair, J., Black, W., Babin, B. y Anderson, R. (2010). Multivariate data analysis: A global perspective. Boston: Pearson Prentice Hall. 
Hall, C. M., Sharples, L., Cambourne, B. y Macionis, N. (2000). Wine tourism around the world: development, management and markets. Oxford: Elsevier.

Hall, M. C., Sharples, L., Mitchell, R., Macionis, N. y Cambourne, B. (2003). Food tourism around the World. Oxford: Butterworth-Heinemann.

Hollow, J., Jones, S. y Taylor, B. (2014). Making sense of urban food festivals: cultural regeneration, disorder and hospitable cities. Journal of Policy Research in Tourism, Leisure \& Events, 6(1), 1-14.

Haven-Tang, C. y Jones, E. (2005). Using local food and drink to differentiate tourism detinations through a sense of place: A stoy from Wales-Dining ar Mommouthshire's Great Table. Journal of Culinary Science and Technology, 4(4), 69-86.

Henderson, J. C. (2009). Food tourism reviewed. British Food Journal,111(4), 317-326.

Hjalager, A.M. (2004). What do tourists eat and why?. Towards a sociology of gastronomy and tourism. Tourism, 52(2), 195-201.

Hjalager, A. - M. y Richards, G. (2002). Tourism and Gastronomy. Londres: Routledge

Hollows, J., Jones, S. y Taylor, B. (2014). Making sense of urban food festivals: cultural regeneration, disorder and hospitable cities. Journal of Policy Research in Tourism, Leisure and Events, 6(1), 1-14.

Horng, J.-S., Liu, C.-H., Chou, H.-Y. y Tsai, C.-Y. (2012). Understanding the impact of culinary brand equity and destination familiarity on travel intentions. Tourism Management, 33(4), 815-824.

Horng, J. S., Su, C.-S. y So, S.-I. A. (2013). Segmenting food festival visitors: applying the theory of planned behavior and lifestyle. Journal of Convention and Event Tourism, 14, 193216.

Ignatov, E. y Smith, S. (2006). Segmenting Canadian culinary tourists. Current Issues in Tourism, 9(3), 235-255.

Instituto Nacional de Estadística (2019). Encuesta de Ocupación Hotelera. Madrid: Servicio de Publicaciones del Instituto Nacional de Estadística.

Jiménez Beltrán, J., López-Guzmán, T. y González Santa-Cruz, F. (2016). Gastronomy and tourism: profile and motivation of international tourism in the city of Córdoba, Spain. Journal of Culinary Science \& Technology, 14(4), 350-366.

Kenji, R. (2016). Consuming sumo wrestlers: Taste, commensality, and authenticity in japanese food. Food, Culture \& Society, 19(4), 637-653.

Kim, Y. G., Eves, A. y Scarles, C. (2009). Building a model of local food consumption on trips and holidays: a grounded theory approach. International Journal of Hospitality Management, 28, 423-431.

Kim, Y. G., Eves, A. y Scarles, C. (2013). Empirical verification of a conceptual model of local consumption at a tourist destination. International Journal of Hospitality Management, 33, 484-489. 
Kim, Y.- H., Ducan, J. y Chang, B. W. (2015). Involvement, satisfaction, perceived value, and revisit intention: a case study of a food festival. Journal of Culinary Science and Technology, 13, 133-158.

Kivela, J. y Crotts, J. (2005). Gastronomy tourism: A meaningful travel market segment. Journal of Culinary Science and Technology, 4(2/3), 39-55.

Ko, S., Kang, kang, H. y Lee, M. (2018). An exploration of foreign tourists' perception of korean food tour: a factor-cluster segmentation approach. Asia Pacific Journal of Tourism Research, 23(8), 833-846.

Kruskal, W. H. y Wallis, W. A. (1952). Use of ranks in one-criterion variance analysis. Journal of the American Statistical Association, 47(260), 583-621.

Lee, K.-H. yScott, N. (2015). Food tourism reviewed using the paradigm funnel approach. Journal of Culinary Science \& Technology, 13, 95-115.

Levitt, J. A., Zhang, P, DiPietro, R. B. y Meng, F. (2019). Food tourist segmentation: Attitude, behavioral intentions and travel planning behavior based on food involvement and motivation. International Journal of Hospitality \& Tourism Administration, 20(2), 129155.

Long, L. M. (2004). Culinary tourism: A folkloristic on eating and otherness. En L. M. Long, (Ed.), Culinary Tourism (pp. 20-50). Lexington: The University Press of Kentucky.

López-Guzmán, T., Hernández-Mogollón, J. M. y Di Clemente, E. (2014). Gastronomic tourism as a motor of local and regional development. Regional and Sectorial Economics Studies, 14(1), 95-102.

López-Guzmán, T., Hernández-Mogollón, J. M. y Di Clemente, E. (2016). Culinary travels as new approach for cultural tourism. Turizam, 20(1), 1-11.

López-Guzmán, T., Uribe-Lotero, C.P., Pérez-Gálvez, J.C. y Ríos-Rivera, I. (2017). Gastronomic festivals: attitude, motivation and satisfaction of the tourist. British Food Journal, 119(2), 267-283.

Mann H.B. y Whitney D.R. (1947). On a test of whether one of two random variables is stochastically larger than the other. The Annals of Mathematical Statistics, pp. 50-60.

Marchini, A. Riganelli, C. y Diotallevi, F. (2019). The success factors of food events: The case study of Umbrian extra virgin olive oil. Journal of Food Products Marketing, 22(2), 147 167.

McKercher, B., Okumus, F. y Okumus, B. (2008). Food Tourism as a Viable Market Segment: It's All How You Cook the Numbers!. Journal of Travel \& Tourism Marketing, 25(2), 137148.

Mgonje, J. T., Backman, K. F., Backman, S. J., Moore, D. D. y Hall, J. C. (2016). A structural model to assess international visitors' perceptions about food in Tanzania. Journal of Sustainable Tourism, 25(6), 796-816.

Morales Vallejo, P., Urosa Sanz, B. y Blanco Blanco, A. (2003). Construcción de escalas de actitudes tipo Likert: una guía práctica. Madrid: La Muralla. 
Nam, J.-H. y Lee, T. J. (2011). Foreign travelers' satisfaction with traditional Korean restaurants. International Journal of Hospitality Management, 30(4), 982-989.

Okumus, B., Koseoglu, M.A. y Ma, F. (2018). Food and gastronomy research in tourism and hospitality: A bibliometric analysis. International Journal of Hospitality Management, 73, 64-74.

Organ, K., Koening-Lewis, N., Palmer, A. y Probert, J. (2015). Festivals as agents for behavior change: A study of food festival engagement and subsequent food choices. Tourism Management, 48, 48-99.

Ottenbacher, M. C. y Harrington, R. (2013). A case study of a culinary tourism campaign in Germany: implications for strategy making and successful implementation. Journal of Hospitality and Tourism Research, 37(1), 3-28.

Park, K. (2017). Ethnic foodscapes: Foreign cuisines in the United States. Food, Culture \& Society, 20(3), 365-393.

Park, D. B. y Yoon, Y. S. (2009). Segmentation by motivation in rural tourism: A Korean case study. Tourism Management, 30(1), 99-108.

Pérez-Gálvez, J. C., Jaramillo Granda, M., López-Guzmán, T. y Reinoso Coronel, J. (2017). Local gastronomy, culture and tourist, sustainable cities: the behavior of the American tourist. Sustainable Cities and Society, 32, 64-612.

Pérez-Priego, M. A., García-Moreno, M., Gómez-Casero, G. y Caridad, L. (2019). Segmentation based on the gastronomic motivations of tourists: the case of the Costa del Sol (Spain). Sustainability, 11, 409-418.

Pesonen, J., Komppula, R., Kronenberg, C. y Peters, M. (2011). Understanding the relationship between push and pull motivations in rural tourism. Tourism Review, 66(3), 32-49.

Prayag, G. (2010). Images as pull factors of a tourist destination: A factor-cluster segmentation analysis. Tourism Analysis, 15(2), 213-226.

Prayag, G. y Hosany, S. (2014). When Middle East meets West: Understanding the motives and perceptions of young tourists from United Arab Emirates. Tourism Management, 40, 35-45.

Quan, S. y Wang, N. (2014). Towards a structural model of the tourist experience: an illustration from food experiences in tourism. Tourism Management, 25(3), 297-305.

Remoaldo, P. C., Vareiro, L, Ribeiro, J. C. y Santos, J. F. (2014). Does gender affect visiting a World Heritage Site. Visitor Studies, 17(1), 89-106.

Robinson, R. N. S. Getz, D. y Donilcar, S. (2018). Food tourism subsegments: A data-driven analysis. International Journal of Tourism Research, 20(3), 367-377.

Sheppard, A. G. (1996). The sequence of factor analysis and cluster analysis: Differences in segmentation and dimensionality through the use of raw and factor scores. Tourism Analysis, 1(1), 49-57.

Sims, R. (2010). Putting place of the menu: the negotiation of locality in UK food tourism, from production to consumption. Journal of Rural Studies, 26, 105-115. 
Smith, S. y Costello, C. (2009). Segmenting visitors to a culinary event: motivations, travel behaviour and expenditures. Journal of Hospitality Marketing and Management, 18, 44-67.

Taar, J. (2014). The best culinary experience. Factors that create extraordinary eating episodes. Procedia-Social and Behavioral Sciences, 122, 145-151.

Tiemann, K. (2008). Introduction. Humanity \& Society, 32(1), 1-1

Thompson, M. y Prideaux, B. (2009). Developing a food and wine segmentation and classifying destinations on the basis of their food and wine sectors. Advances in Hospitality and Leisure, 5, 163-183.

Timothy, D J. (Ed.) (2016). Heritage cuisines: traditions, identities and tourism. Abingdon: Routledge.

Timothy, D. J. y Ron, A. S. (2013). Understanding heritage cuisines and tourism: identity, image, authenticity, and change. Journal of Heritage Tourism, 8(2-3), 99-104.

Ting, H., Tan, S. y John, A. N. (2017). Consumption intention towards ethnic food. Determinants of Dayak food choice by Malaysians. Journal of Ethnic Foods, 4(1), 21-27.

Torres Chavarria, L. C. y Phakdee-Auksern, P. (2017). Understanding international tourists' attitudes towards street food in Phuket, Thailand. Tourism Management Perspectives, 21, 66-73.

Tse, P. y Crotts, J. C. (2005). Antecedents of novelty seeking: International visitors propensity to experiment across Hong Kong's culinary traditions. Tourism Management, 26, 965968.

Ukenna, S. I. y Ayadele, A. A. (2019). Applying the extended Theory of Planned Beahavior to predict sustainable Street food patronage in a Developing Economy. Journal of Food Product Marketing, 25(4), 404-434.

UNWTO (2012). Global report on food tourism. Madrid: Servicio de Publicaciones de la Organización Mundial del Turismo.

UNWTO (2017). Second Global report on food tourism. Madrid: Servicio de Publicaciones de la Organización Mundial del Turismo.

Wu, H.- C., Wong, J. X. - C. y C.- C. Cheng (2014). An empirical study of behavioural intentions in the food festival. The case of Macau. Asia Pacific Journal of tourism Research, 19(11), 1278-1305.

\section{CONTRIBUCIONES DE LOS AUTORES:}

Autor 1: Conceptualización y aprobación de la versión final.

Autor 2: Conceptualización y Análisis, adquisición e interpretación de datos.

Autor 3: Análisis, adquisición e interpretación de datos y redacción y revisión crítica.

Autor 4: Redacción y revisión crítica y aprobación versión final. 